\title{
Domain decomposition with Robin boundary conditions across a phase interface
}

\author{
Hugh M. Blackburn* \\ (Received 7 August 2000)
}

\begin{abstract}
Domain decompositions are typically pursued in order to split up large elliptic boundary-value problems into a set of smaller problems that can each be solved separately, with the solution to the larger problem obtained through iterative application of inter-domain boundary conditions. Here we review a related problem that arises in the study of unsteady advection-diffusion with two solvent phases. A boundary
\end{abstract}

* CSIRO Building Construction \& Engineering, PO Box 56, Highett, Vic 3190, Australia. mailto:hugh.blackburn@dbce.csiro.au

${ }^{0}$ See http://anziamj . austms . org. au/V42/CTAC99/Blac for this article and ancillary services, (C) Austral. Mathematical Soc. 2000. Published 27 Nov 2000. 
iteration scheme is devised and tested within the context of an idealised Poisson problem. The method is then used in the simulation of capillary chromatography and the results compared to an available analytical solution.

\section{Contents}

1 Introduction

C265

2 Physical description

C266

2.1 Flow field . . . . . . . . . . . . . . C267

2.2 Solute transport . . . . . . . . . . . . . C267

2.3 Boundary conditions at the phase interface . . . . . . . C269

3 Discretisation

$\mathrm{C} 270$

3.1 Fourier expansions ................ . . C270

3.2 Temporal evolution . . . . . . . . . . . . . . . . C272

3.3 Galerkin spectral element method . . . . . . . . C C273

4 Iterative coupling

C275

5 Analytical solutions for solute transport $\quad$ C277

$\begin{array}{lll}6 & \text { Simulation results } & \text { C281 }\end{array}$

6.1 Numerical convergence . . . . . . . . . . . . . . C283 
6.2 Mass conservation . . . . . . . . . . . . . . C287

6.3 Peak transport and broadening rates . . . . . . . . C287

7 Conclusions

C289

References

C289

\section{Introduction}

The problem of transport of solute in a laminar flow within a coated tube of circular cross section - a capillary separation column - was studied by Aris [1], who provided analytical solutions for the transport and dispersion rates. Aris also gave an analytical solution for the case where both the tube and the coating are of arbitrary (but axially constant) section, with the restriction that one phase must completely surround the other; in topological terms, the outer, 'stationary', phase must be doubly connected, the inner, 'mobile', phase can be either simply or doubly connected. In order to remove these restrictions a numerical solution of the problem has been pursued.

At the interface between the mobile solute phase and the stationary coating phase, Robin mass-transfer conditions apply that result in coupling of unsteady transport in the two domains. The tendency towards unequal static partition of solute species between the two phases and the properties of the interphase coupling results in the separation of different solute species; var- 
ious species are transported by the flow at different rates, as they spend differing average amounts of time in the stationary phase.

We describe the physical problem to be studied and show that its discrete analogue produces a set of Helmholtz equations that are coupled through the Robin interphase boundary conditions. Two iterative methods for computing the coupling are studied in the simplified setting of coupled two-dimensional Poisson equations. Finally we demonstrate that the numerical method for the advection-diffusion problem successfully simulates the transport and dispersion of solute within the column.

\section{Physical description}

The separation column is decomposed into two domains, one for the mobile phase, and one for the stationary phase, as illustrated in Figure 1 for a column of rectangular section. Inert carrier gas flows within the mobile phase domain, and it is assumed that the transport of the carrier and the solute are uncoupled. The approach taken simulates transport over a length of tube for which the pressure drop is minimal compared to the mean pressure; within this length (e.g. 1000 tube diameters), the flow of carrier gas can be considered incompressible. The tube diameter is assumed to be sufficiently large compared to the molecular mean free path that all species can be considered to be continua. (For a non-circular cross-section we define an equivalent diameter as $(4 A / \pi)^{1 / 2}$, where $A$ is the cross-sectional area.) The Reynolds 
number of the flow is low enough for the flow to be laminar.

\subsection{Flow field}

The flow of carrier gas is laminar, steady, and uniform in the z-direction. Under these assumptions, the Navier-Stokes equations for the fluid flow reduce to a two-dimensional Poisson equation for the single velocity component $w(x, y)$

$$
\nabla^{2} w=\frac{1}{\mu}\left(-\frac{\mathrm{d} p}{\mathrm{~d} z}\right) \quad \text { on } \Omega_{M},
$$

where $p$ is the pressure and $\mu$ is the dynamic viscosity of the carrier gas. Given the applied pressure gradient and viscosity, (1) is solved for $w$ in the mobile phase domain, $\Omega_{M}$, with boundary condition $w=0$ at the wall (here, the $\Omega_{M}-\Omega_{S}$ phase interface, $\Gamma$ ).

\subsection{Solute transport}

Transport for the concentration, $c$, of a sample species in the stationary phase is governed by the unsteady diffusion equation

$$
\frac{\partial c}{\partial t}=D_{S} \nabla^{2} c \quad \text { on } \Omega_{S}
$$




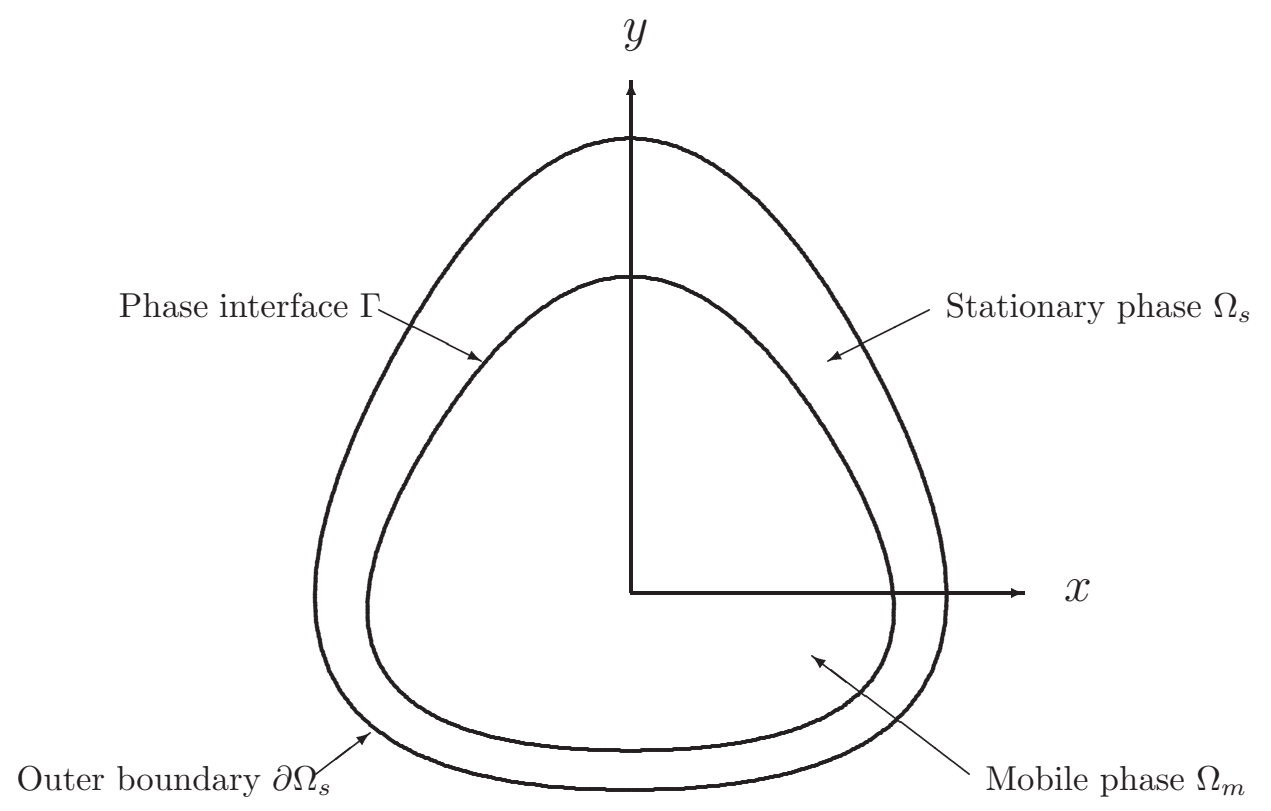

FiguRE 1: Diagram showing the cross-section of a separation column of arbitrary shape; the column is uniform in the $z$ (out-of-page) direction, which is the direction of carrier gas flow. 
where $D_{S}$ is the diffusion coefficient for species $c$ in the stationary phase. In the mobile phase, transport of $c$ is governed by the unsteady advectiondiffusion equation

$$
\frac{\partial c}{\partial t}+w \frac{\partial c}{\partial z}=D_{M} \nabla^{2} c \quad \text { on } \Omega_{M}
$$

where $w$ is the gas phase velocity in the mobile phase, and $D_{M}$ is the diffusion coefficient for species $c$ in the carrier gas.

The outer wall of the column $\left(\delta \Omega_{S}\right)$ is assumed inert and impervious to $c$, so the appropriate boundary condition to be applied at $\delta \Omega_{S}$ is zero flux, i.e. $\partial c / \partial n=0$, where $\partial / \partial n$ is a derivative taken in the outward normal direction. Where domain symmetry can be used to simplify the problem, the appropriate boundary condition is again specification of zero flux at the symmetry plane.

\subsection{Boundary conditions at the phase interface}

At the phase interface $\Gamma$, special boundary conditions must be applied. These have to account for the partition coefficient $K_{c}$, which expresses the equilibrium partition of concentration $c$ between the stationary and mobile phases; $c_{S}=K_{c} c_{M}$ in equilibrium. In addition, they have to account for equality of fluxes of $c$ on each side of the interface, which in turn are proportional to the disequilibrium $c_{S}-K_{c} c_{M}$. The appropriate conditions are [1]

$$
D_{M} \frac{\partial c_{M}}{\partial n}=-D_{S} \frac{\partial c_{S}}{\partial n}=K_{m}\left(c_{S}-K_{c} c_{M}\right)
$$


where the directions of the unit outward normals, $\boldsymbol{n}$, are equal and opposite at the interface $\Gamma$ between $\Omega_{M}$ and $\Omega_{S}$. Variation of the partition coefficient $K_{c}$ between solute species is typically the primary determinant of species separation rates, although the diffusion coefficients can also have a strong influence.

The matching condition (4) can be rearranged to give a pair of Robin boundary conditions that are applied simultaneously in $\Omega_{M}$ and $\Omega_{S}$ :

$$
\begin{aligned}
& \frac{\partial c_{M}}{\partial n}+\frac{K_{m} K_{c}}{D_{M}}\left(c_{M}-c_{S} / K_{c}\right)=0 \text { on } \Omega_{M}, \\
& \frac{\partial c_{S}}{\partial n}+\frac{K_{m}}{D_{S}}\left(c_{S}-K_{c} c_{M}\right)=0 \text { on } \Omega_{S} .
\end{aligned}
$$

These have the generic form

$$
\frac{\partial c}{\partial n}+K(c-C)=0
$$

\section{Discretisation}

\subsection{Fourier expansions}

As the column is uniform in the axial $(z)$ direction, it is advantageous to assume that the problem is periodic in that direction, with periodic length 
$L_{z}$ :

$$
c(x, y, z, t)=c\left(x, y, z+L_{z}, t\right) .
$$

A slug of high concentration advected down the tube will seem to pass out one end and return through the other. Provided the axial length scale of the slug is always much shorter than the length of tube simulated, the results will well represent the actual transport.

With the assumption of periodicity, the three-dimensional scalar field $c$ can be projected exactly onto a set of two-dimensional complex Fourier modes

$$
\hat{c}_{k}(x, y, t)=\frac{1}{L_{z}} \int_{0}^{L_{z}} c(x, y, z, t) e^{-i \beta k z} \mathrm{~d} z
$$

with the Fourier series reconstruction

$$
c(x, y, z, t)=\sum_{k=-\infty}^{+\infty} \hat{c}_{k}(x, y, t) e^{i \beta k z},
$$

where $i=(-1)^{1 / 2}$ and $\beta=2 \pi / L_{z}$. For numerical approximation, the set of modes is truncated at some finite number $M$; if the solution is smooth in the $z$-direction, the truncated approximation converges to the solution exponentially fast [2]. After Fourier transformation, the equivalents of the gradient and Laplacian operators become

$$
\tilde{\nabla} \equiv(\partial / \partial x, \partial / \partial y, i \beta k), \quad \tilde{\nabla}^{2} \equiv\left(\partial^{2} / \partial x^{2}, \partial^{2} / \partial y^{2},-\beta^{2} k^{2}\right) \equiv \tilde{\nabla}_{x y}^{2}-\beta^{2} k^{2}
$$

and transport equations (2) and (3) become

$$
\frac{\partial \hat{c}_{k}}{\partial t}=D_{S} \tilde{\nabla}^{2} \hat{c}_{k} \quad \text { on } \Omega_{S}
$$




$$
\frac{\partial \hat{c}_{k}}{\partial t}+i \beta k w \hat{c}_{k}=D_{M} \tilde{\nabla}^{2} \hat{c}_{k} \text { on } \Omega_{M}
$$

for each Fourier mode $k$. Owing to the linearity of the original equations, Fourier transformation completely decouples the various modes, which can be evolved independently and/or concurrently. Thus the originally threedimensional problem is converted into a set of two-dimensional problems.

\subsection{Temporal evolution}

A mixed explicit-implicit temporal evolution approximation is applied to the transformed transport equations; a fully implicit scheme could be implemented, but at the expense of requiring complex arithmetic. The temporal derivative of $\hat{c}_{k}$ is approximated at time level $n+1$ using a backwarddifferencing scheme of order $J$

$$
\frac{\partial \hat{c}_{k}^{(n+1)}}{\partial t} \approx \frac{1}{\Delta t}\left(\gamma_{0} \hat{c}_{k}^{(n+1)}+\sum_{q=0}^{J-1} \alpha_{q} \hat{c}_{k}^{(n-q)}\right)
$$

while the advective terms are approximated at time level $n+1$ using polynomial extrapolation of order $J$

$$
i \beta k w \hat{c}_{k}^{(n+1)} \approx \beta k w \sum_{q=0}^{J-1} \rho_{q} i \hat{c}_{k}^{(n-q)} .
$$

The discrete weights $\gamma_{0}, \alpha_{q}, \rho_{q}$ for schemes up to third order have been tabulated by Karniadakis et al. [3]. Applying these approximations produces 
the following semi-discrete Helmholtz equations for the evolution of each Fourier mode

$$
\begin{gathered}
\left(\tilde{\nabla}_{x y}^{2}-\frac{\gamma_{0}}{D_{S} \Delta t}+\beta^{2} k^{2}\right) \hat{c}_{k}^{(n+1)}=\frac{1}{\Delta t D_{S}} \sum_{q=0}^{J-1} \alpha_{q} \hat{c}_{k}^{(n-q)} \text { on } \Omega_{S} \\
\left(\tilde{\nabla}_{x y}^{2}-\frac{\gamma_{0}}{D_{M} \Delta t}+\beta^{2} k^{2}\right) \hat{c}_{k}^{(n+1)}=\frac{\beta k w}{D_{M}} \sum_{q=0}^{J-1} \rho_{q} i \hat{c}_{k}^{(n-q)}+\frac{1}{\Delta t D_{M}} \sum_{q=0}^{J-1} \alpha_{q} \hat{c}_{k}^{(n-q)}
\end{gathered}
$$

on $\Omega_{M}$, or in generalised form

$$
\left(\tilde{\nabla}_{x y}^{2}-\lambda_{k}^{2}\right) \hat{c}_{k}=\hat{f}_{k}
$$

At this stage the overall structure of the discrete problem is apparent: at each timestep there are two elliptic (Helmholtz) problems to be solved, coupled through Robin boundary conditions at the phase interface. In order to further define the discretisation we must adopt a numerical method for solution of the elliptic problems.

\subsection{Galerkin spectral element method}

To complete the discretisation, a Galerkin spectral element method is applied to the modal Helmholtz equations (15), as well as to the Poisson problem (1) for the velocity field $w$. To arrive at the Galerkin formulation, the partial 
differential equation is first multiplied by a weight or test function $v$ and integrated over the domain, following which the integral is symmetrised by applying Gauss' theorem. This procedure results in the so-called weak form of the original equation. Applied to (15) this produces

$$
\int_{\Omega}\left(\tilde{\nabla}_{x y} \hat{c}_{k} \cdot \tilde{\nabla}_{x y} v+\lambda_{k}^{2} \hat{c}_{k} v\right) \mathrm{d} \Omega=-\int_{\Omega} \hat{f}_{k} v \mathrm{~d} \Omega+\int_{\Gamma} \frac{\partial \hat{c}_{k}}{\partial n} v \mathrm{~d} \Gamma,
$$

where $\Omega$ is either $\Omega_{M}$ or $\Omega_{S}$, and $\Gamma$ represents any boundary on which $\hat{c}_{k}$ is not directly specified, i.e. all boundaries in the present problem. One means of accommodating Robin boundary conditions (6) on $\Gamma$ is to insert them into the last term in (16), and then to rearrange the result to give

$$
\int_{\Omega}\left(\tilde{\nabla}_{x y} \hat{c}_{k} \cdot \tilde{\nabla}_{x y} v+\lambda_{k}^{2} \hat{c}_{k} v\right) \mathrm{d} \Omega+\int_{\Gamma} K \hat{c}_{k} v \mathrm{~d} \Gamma=-\int_{\Omega} \hat{f}_{k} v \mathrm{~d} \Omega+\int_{\Gamma} K C v \mathrm{~d} \Gamma .
$$

A Galerkin problem results when the function space from which trial functions $\hat{c}_{k}$ and test functions $v$ are drawn is the same. This must be a Sobolev-1 space for (17) to be guaranteed convergence [7]. For discrete solution a finite number of functions are chosen, leading to a system of algebraic equations. For solution in complex geometries, the domain is subdivided into a set of simpler geometries (finite elements), with the test and trial functions having compact support over adjoining elements. Typically the integrals in (17) are approximated numerically using an appropriate Gauss rule.

The method is further defined by choosing a function space from which the finite element test and trial functions are drawn. The conventional finite 
element method uses functions which are typically low-order polynomials. The spectral element method [5] uses the same techniques as the conventional finite element method but employs functions constructed from high order orthogonal polynomials (e.g. Legendre polynomials) that satisfy a singular Sturm-Liouville problem. For problems with smooth solutions, the spectral element method generates approximations that converge exponentially to the true solution as the polynomial order in each element is increased [4].

\section{Iterative coupling}

Coupling of the two domains can be accomplished iteratively. Techniques developed for interdomain coupling in elliptic problems discussed in [6] are designed for the case where there is a single global problem (e.g. with identical mechanical properties in each sub-domain) and also are not directly applicable for domains coupled through Robin boundary conditions.

Two techniques were devised and tested in a simplified setting - a Poisson equation $\nabla^{2} c=-2$ imposed on two domains covering the unit square: $\Omega_{M}=$ $[0,1] \times[0,0.9]$, made up of four elements, $\Omega_{S}=[0,1] \times[0.9,1]$, made up of two elements. Homogeneous Dirichlet boundary conditions were imposed around the periphery of the square, while over the interface $(0,0.9)-(1,0.9)$

$$
\frac{\partial c_{M}}{\partial n}+100\left[c_{M}-0.1 c_{S}\right]=0 \quad \text { on } \Omega_{M}
$$




$$
\frac{\partial c_{S}}{\partial n}+1000\left[c_{S}-10 c_{M}\right]=0 \text { on } \Omega_{S}
$$

One iteration technique is to treat (18) as Neumann boundary conditions, using values of $c_{M}$ and $c_{S}$ obtained on previous iterations as data, so that on iteration $(n)$

$$
\frac{\partial c_{M}^{(n)}}{\partial n}=100\left[0.1 c_{S}^{(n-1)}-c_{M}^{(n-1)}\right], \quad \frac{\partial c_{S}^{(n)}}{\partial n}=1000\left[10 c_{M}^{(n-1)}-c_{S}^{(n-1)}\right] .
$$

In the Galerkin form of the discretised equations, these boundary terms contribute only to the forcing. This iteration technique was found to be unstable, even with under-relaxation.

An alternative is to interpret the boundary conditions as of Robin type, so that in each sub-domain only the values of $c$ from the other side of the interface are treated as data supplied by the previous iteration:

$$
\frac{\partial c_{M}^{(n)}}{\partial n}=100\left[0.1 c_{S}^{(n-1)}-c_{M}^{(n)}\right], \quad \frac{\partial c_{S}^{(n)}}{\partial n}=1000\left[10 c_{M}^{(n-1)}-c_{S}^{(n)}\right] .
$$

In this case, as indicated by (17), the values at step $(n)$ are interpreted as contributing to the system stiffness matrix, while those at $(n-1)$ contribute to the forcing. This iteration technique was found to be convergent, and was adopted for the remainder of the study. Iteration continues until the relative change in the $L_{2}$-norm of the solution in each domain drops below a fixed tolerance $\epsilon$. In the unsteady advection-diffusion problem, this sub-iteration occurs every time step. 
To illustrate the solutions obtained for the model problem, a contour plot of the value of $c$ is shown in Figure 2, which also shows element boundaries, and the values of $c$ extracted along the line $(0.5,0)-(0.5,1)$ are shown in Figure 3. Values of $c$ are discontinuous over the interface at $y=0.9$, and it can be verified that the values and slopes satisfy (18). A convergence tolerance $\epsilon=1 \times 10^{-8}$ was employed.

\section{Analytical solutions for solute transport}

Aris [1] provided detailed solutions for the case in which the cross-sectional boundaries of all phases are concentric circles, with the mobile phase lying between radii $r_{0}$ and $r_{1}$, the stationary phase between $r_{1}$ and $r_{2}$. He was able to show that the location of the first moment (mean) of the concentration peak travels with speed

$$
V=\frac{\bar{w}_{1}\left(r_{1}^{2}-r_{0}^{2}\right)+\bar{w}_{2} K_{c}\left(r_{2}^{2}-r_{1}^{2}\right)}{\left(r_{1}^{2}-r_{0}^{2}\right)+K_{c}\left(r_{2}^{2}-r_{1}^{2}\right)}
$$

where $\bar{w}_{1}$ is the mean velocity in the inner phase, $\bar{w}_{2}$ the mean velocity in the outer phase. In the usual case where $r_{0}=0$ and $\bar{w}_{2}=0$, this reduces to the standard result

$$
V=\bar{w}_{1} \frac{r_{1}^{2}}{r_{1}^{2}+K_{c}\left(r_{2}^{2}-r_{1}^{2}\right)}=\bar{w}_{1} \frac{1}{1+k},
$$




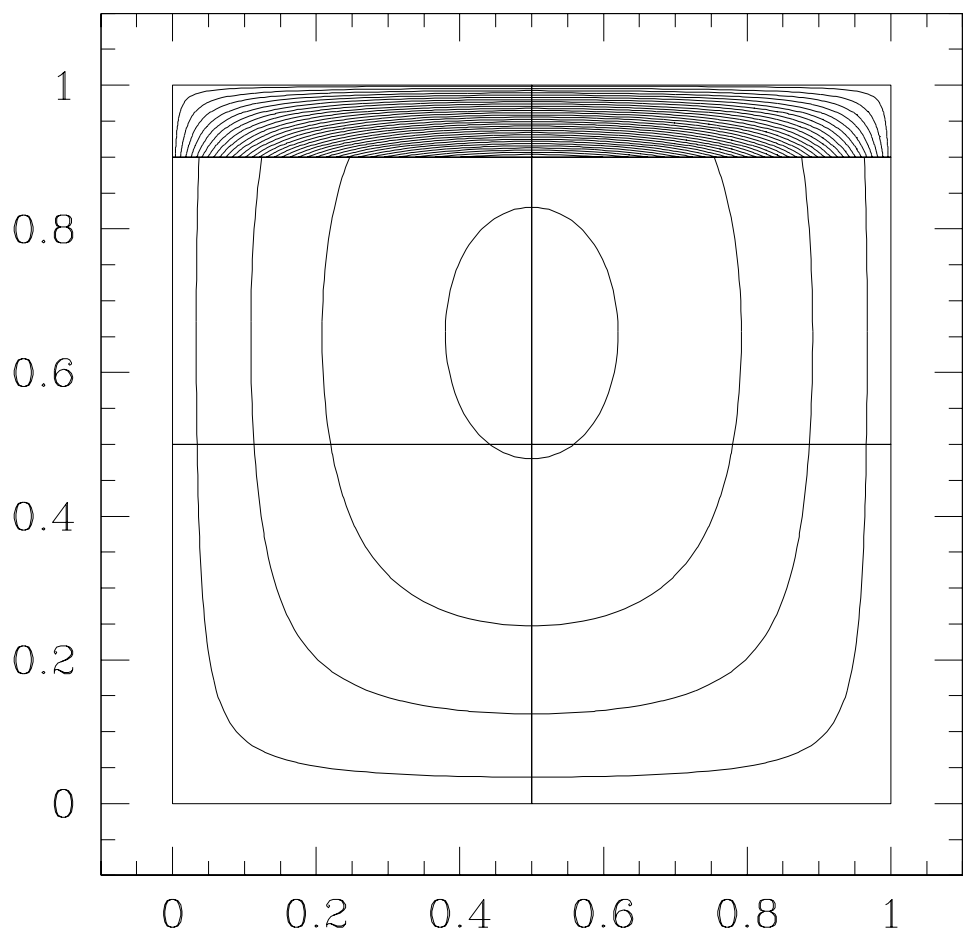

Figure 2: Contour plot of $c$ for solution of a Poisson equation, with a mixed boundary condition patch at $(0,0.9)-(1,0.9)$. Straight lines show locations of element boundaries. 


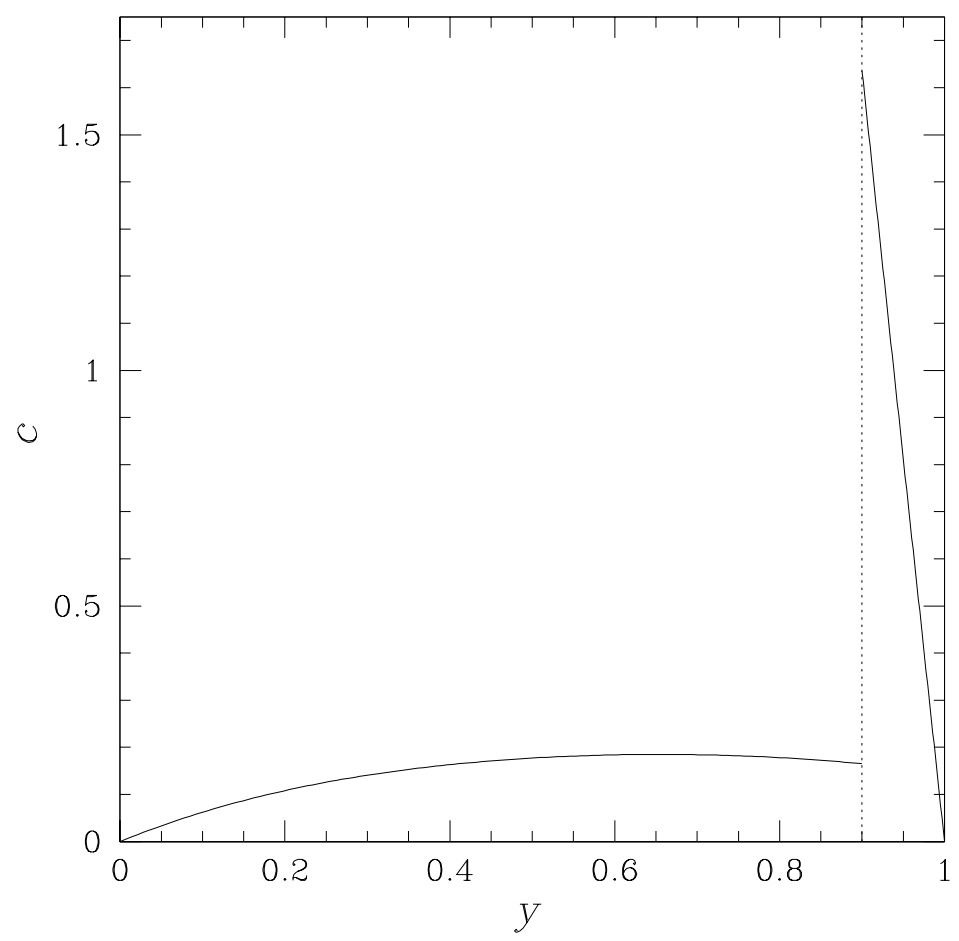

Figure 3: Values of $c$ obtained along a vertical centreline traverse of the two domains in Figure 2. 
where $k$ is the 'retention factor' $K_{c} / \beta$; in turn the 'phase ratio' $\beta=r_{1}^{2} /\left(r_{2}^{2}-\right.$ $\left.r_{1}^{2}\right)$. Aris [1] also provided equations for the time rate of change of the higher moments of the (radially-integrated) concentration profile, for example of the variance $\sigma^{2}$, i.e. the peak-broadening rate. Here Aris' equation (17) is simplified for the case $r_{0}=\bar{w}_{2}=0$ :

$$
\begin{aligned}
\frac{\mathrm{d} \sigma^{2}}{\mathrm{dt}}=\frac{2}{1+k}\left\{D_{M}+\kappa_{1} \frac{\bar{w}_{1}^{2} r_{1}^{2}}{D_{M}}\right\}+\frac{2 k}{1+k}\left\{D_{S}+\kappa_{2} \frac{\bar{w}_{1}^{2}\left(r_{2}^{2}-r_{1}^{2}\right)}{D_{S}}\right\}+ \\
+\frac{k^{2}}{(1+k)^{3}} \frac{r_{1} \bar{w}_{1}^{2}}{K_{m} K_{c}}
\end{aligned}
$$

where, with $\rho=r_{2} / r_{1}$

$$
\kappa_{1}=\frac{1+6 k+11 k^{2}}{48(1+k)^{2}}, \quad \kappa_{2}=\frac{2 \rho^{4} \ln \rho^{2} /\left(\rho^{2}-1\right)-3 \rho^{2}+1}{8(1+k)^{2}\left(\rho^{2}-1\right)} .
$$

In the mobile phase, domain radius $r_{1}$, the carrier phase velocity at any radius and its mean value are respectively

$$
w(r)=\frac{r_{1}^{2}-r^{2}}{4 \mu}\left(-\frac{\mathrm{d} p}{\mathrm{~d} z}\right), \quad \bar{w}=\int_{\Omega_{M}} w \mathrm{~d} \Omega / \int_{\Omega_{M}} \mathrm{~d} \Omega=\frac{r_{1}^{2}}{8 \mu}\left(-\frac{\mathrm{d} p}{\mathrm{~d} z}\right) .
$$

More generally, $w$ is obtained through solution of (1). 


\section{Simulation results}

In order to test the numerical method developed for the coupled advectiondiffusion problem, the time rate of change of the location and spread of the concentration peak compared to the analytical results (21) and (22) for one set of parameters. The mobile phase region was $250 \mu \mathrm{m}$ diameter, with a $1 \mu \mathrm{m}$ thick stationary phase coating. Spectral element meshes for the two domains are illustrated in Figure 4; symmetry properties allow only a quarter of the domain to be represented.

The flow in the column was set to have a mean velocity of $1 \mathrm{~m} / \mathrm{s}$ : for laminar flow in a circular tube, the analytical result (24) for the velocity profile is a parabola of revolution. The computed result for the velocity profile with the analytical value of $(d p / d z) / \mu$ had a maximum error of order $10^{-8}$, with 9 th order shape functions used in the spectral elements.

Diffusion coefficients were given representative values of $D_{M}=40 \times$ $10^{-6} \mathrm{~m}^{2} / \mathrm{s}, D_{S}=10 \times 10^{-9} \mathrm{~m}^{2} / \mathrm{s}$. The partition coefficient was set as $K_{c}=10$, and the mass transfer coefficient was chosen as $K_{m}=1 \mathrm{~m} / \mathrm{s}$. Given these values, (21) predicts a mean transport velocity of $0.861594 \mathrm{~m} / \mathrm{s}$, and from (22), $\mathrm{d} \sigma^{2} / \mathrm{d} t=92.3263 \times 10^{-6}+6.87980 \times 10^{-6}+0.206312 \times 10^{-6}=99.4124 \times$ $10^{-6} \mathrm{~m}^{2} / \mathrm{s}$, showing the relative contributions of the three terms.

The length of tube used in the simulations was $L_{z}=0.2 \mathrm{~m}$, with 64 data planes (32 Fourier modes) in the $z$-direction. An initial condition of a Gaussian-shaped concentration pulse was set in $\Omega_{M}$, centred on the centre 


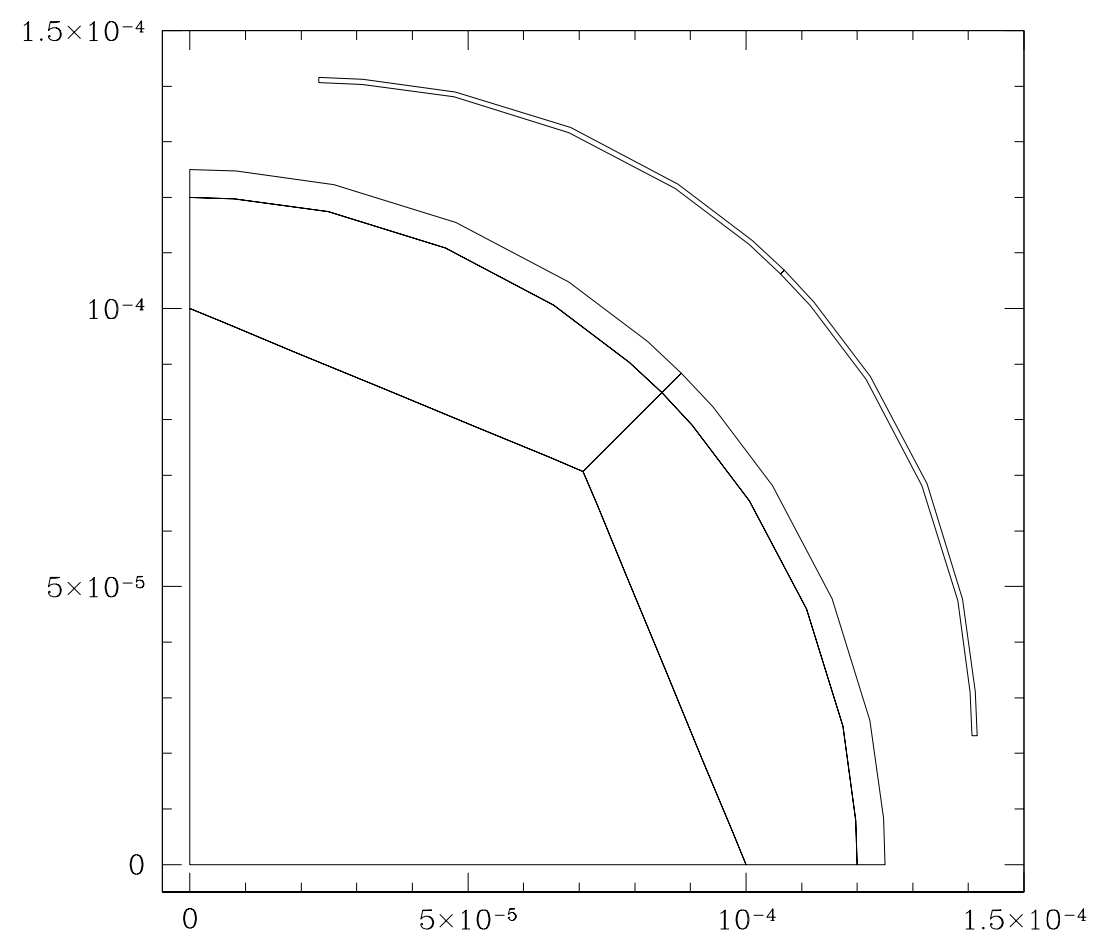

Figure 4: Two-domain spectral element mesh for simulation of transport in a separation column of circular section. There are five elements in the mobile phase and two in the stationary phase - the two phases are shown separated for clarity. 
point of the pipe, with a peak concentration of 1 (arbitrary units) and an initial longitudinal variance $\sigma^{2}=32 \times 10^{-6} \mathrm{~m}^{2}$. The simulation was run for a time of $0.1 \mathrm{~s}$ (4000 timesteps of $0.000025 \mathrm{~s}$ ), with a second-order time-stepping scheme, i.e. $J=2$ in (13) and (14). In the absence of the stationary phase, the mean gas velocity would advect the concentration peak to the end of the domain in the duration of the simulation.

Profiles of tube centreline concentration are shown in Figure 5 starting at the initial condition, then at four times up to $t=0.1 \mathrm{~s}$. The decline from the initial peak is brought about by a combination of radial equilibration and partition into the stationary phase of the initially Gaussian-shaped concentration pulse. The effects of domain periodicity may just be observed for the final profile. Clearly the partition between phases has the expected effect of slowing the mean transport rate.

\subsection{Numerical convergence}

A number of variables contribute to the numerical convergence properties of the solution. These include (i) the number and position of spectral elements; (ii) polynomial order of the shape functions used within each element; (iii) tolerance set on iterative coupling of mobile and stationary phase solutions; (iv) the number of data planes in the $z$-direction; (v) time stepping order; (vi) time step size. For the test problem defined above the most significant variables are items (iii) and (ii). The effects of these variables are demonstrated 


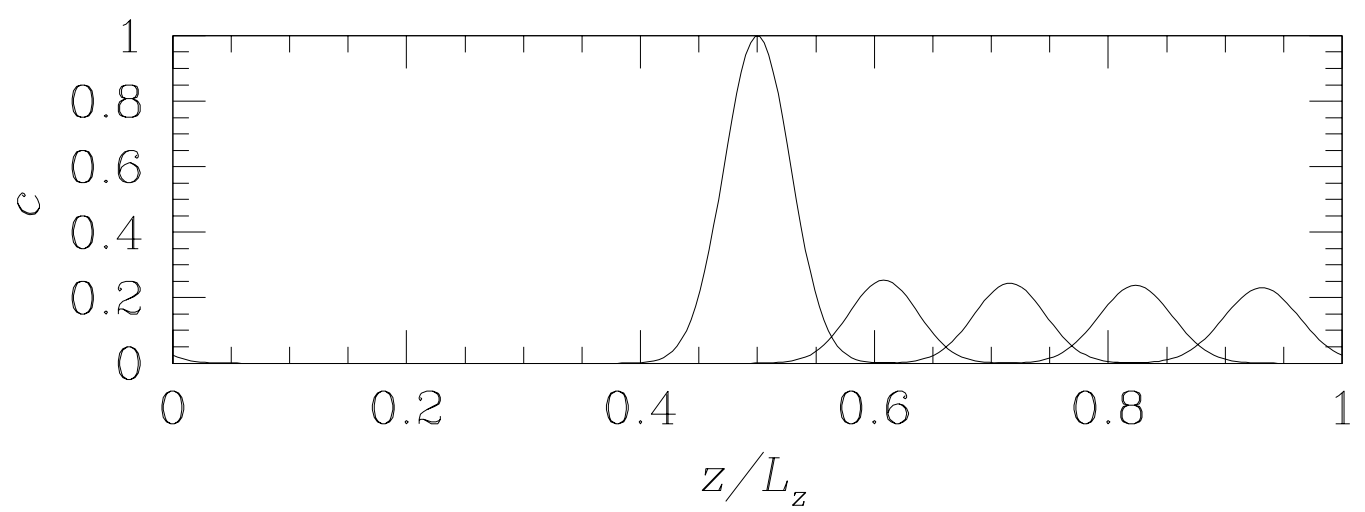

Figure 5: Tube centreline concentration profiles for flow in a column of circular cross section. Partition coefficient $K_{c}=10$. Profiles are for times $t=0.0 \mathrm{~s}, 0.025 \mathrm{~s}, 0.05 \mathrm{~s}, 0.075 \mathrm{~s}, 0.1 \mathrm{~s}$. 
by examining the convergence of the position and spread of the concentration peak. These are assessed using cross-sectional integrals of concentration in the mobile phase. The mean and variance of the concentration profiles are approximated numerically as

$$
\begin{gathered}
\mu=\int_{0}^{L_{z}} z C(z) \mathrm{d} z / \int_{0}^{L_{z}} C(z) \mathrm{d} z \approx \sum_{j=0}^{N_{z}-1} z_{j} C_{j} / \sum_{j=0}^{N_{z}-1} C_{j} \\
\sigma^{2}=\int_{0}^{L_{z}}(z-\mu)^{2} C(z) \mathrm{d} z / \int_{0}^{L_{z}} C(z) \mathrm{d} z \approx \sum_{j=0}^{N_{z}-1}\left(z_{j}-\mu\right)^{2} C_{j} / \sum_{j=0}^{N_{z}-1} C_{j},
\end{gathered}
$$

where $C(z)=\int_{\Omega_{M}} c(z) \mathrm{d} \Omega$ is the cross-sectional integral of $c$ at location $z$, computed using Gauss-Lobatto quadrature. Higher moments of the concentration profile may be similarly approximated.

As an example of an examination of convergence, consider the effect of changing the iterative coupling tolerance $\epsilon$ at a fixed spectral element polynomial order $p=9$, as shown in Figure 6 . The results appear to converge satisfactorily at $\epsilon \approx 10^{-8}$. Similar effects are observed in plots of $\sigma^{2}$ versus $t$.

Other investigations have shown that variations in spectral element polynomial order $p$ have little observable effect on $\mu$ and $\sigma^{2}$, within the range $p=5-9$. Likewise for variations in timestep $\Delta t=0.000025,0.00005,0.0001$. However, readily observable effects are apparent in the higher moments of the concentration profile. 


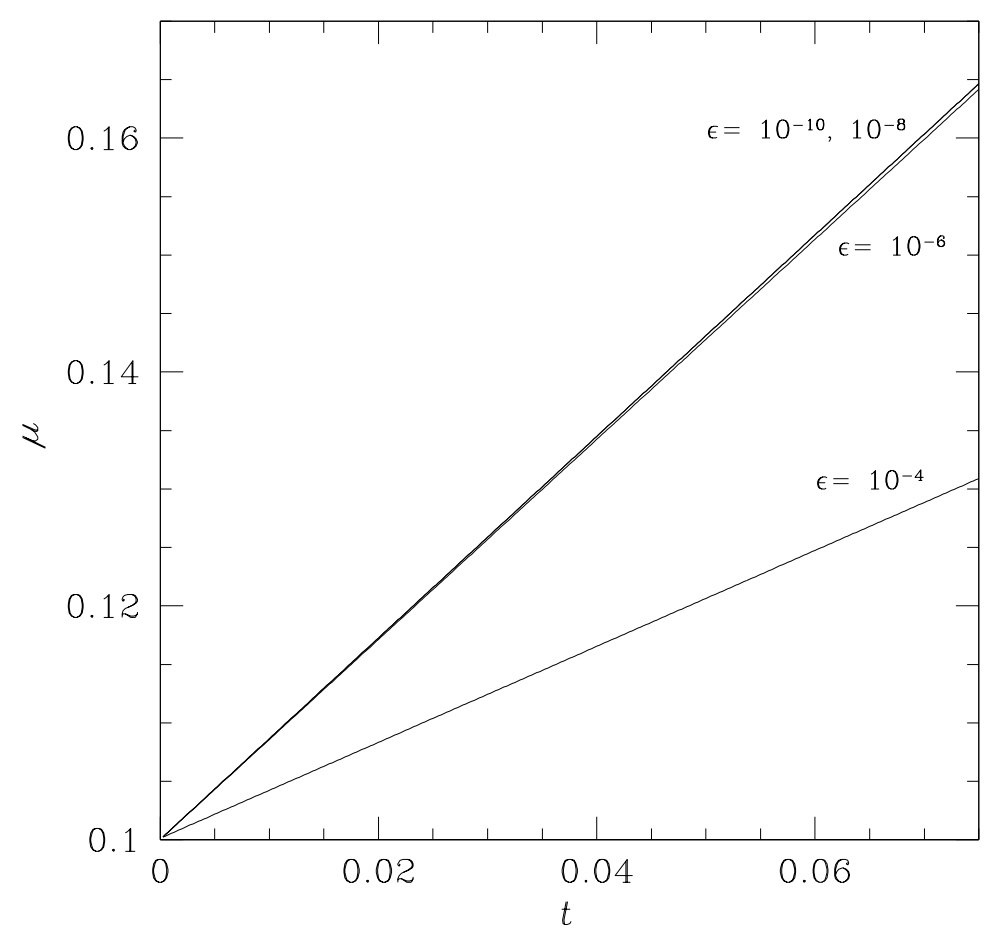

Figure 6: Plot showing the effect of iterative coupling tolerance $\epsilon$ on mean transport rate. 


\subsection{Mass conservation}

The total mass of the solute should be conserved. At initial conditions, all of the solute species resides in the mobile phase, but the distribution rapidly evolves towards equilibrium partition, so that the ratio of the amounts in the phases (stationary/mobile) is $K_{c} / \beta$, while the total amount remains constant. Figure 7 illustrates this rapid redistribution process. From the numerical results at $t=0.1 \mathrm{~s}$, the ratio of total amount of $c$ in the two phases is 0.16063 , compared to $K_{c} / \beta=0.16064$.

\subsection{Peak transport and broadening rates}

Following an initial transient phase lasting approximately $0.05 \mathrm{~s}$, the time rates of change of $\mu$ and $\sigma^{2}$ are observed to be approximately constant. Values extracted from solutions at $t=0.05$ and $t=0.075$ give

$$
\begin{gathered}
\frac{\mathrm{d} \mu}{\mathrm{d} t} \approx \frac{0.1646527-0.1431127}{0.025}=0.861599 \mathrm{~m} / \mathrm{s} \\
\frac{\mathrm{d} \sigma^{2}}{\mathrm{~d} t} \approx \frac{39.45421 \times 10^{-6}-36.96894 \times 10^{-6}}{0.025}=99.4109 \times 10^{-6} \mathrm{~m}^{2} / \mathrm{s}
\end{gathered}
$$

which compare very favourably to the analytical values of $0.861594 \mathrm{~m} / \mathrm{s}$ and $99.4124 \times 10^{-6} \mathrm{~m}^{2} / \mathrm{s}$ given by (21) and (22) respectively. 


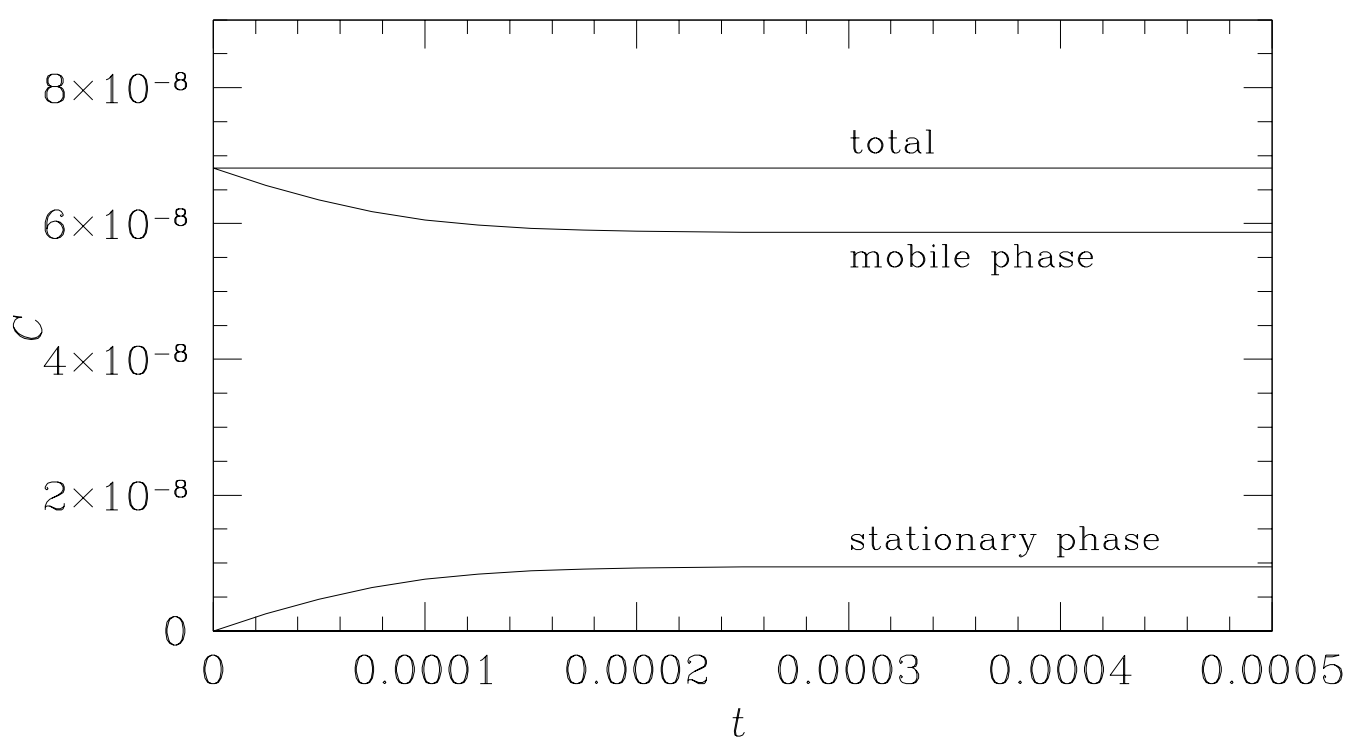

FiguRE 7: Plot showing evolution of the partition of the volume integral of the concentration with time. 


\section{Conclusions}

Starting from physical fundamentals, a numerical simulation method was developed for simulation of species transport in capillary chromatography. This required development of a new iterative technique for domain decomposition coupled across a phase boundary through Robin boundary conditions. Results demonstrate the success of the coupling method, and its application to predicting mean transport and peak-broadening rates in capillary separation.

Acknowledgement: this work was supported by AusIndustry $R \& D$ Start Grant GRA 00621 to SGE International Pty Ltd.

\section{References}

[1] R. Aris. On the dispersion of a solute by diffusion, convection and exchange between phases. Phil. Trans. Roy. Soc. Lond. A, 252:538-550, 1959. C265, C269, C277, C280

[2] D. Gottlieb and S. A. Orszag. Numerical Analysis of Spectral Methods: Theory and Applications. SIAM, Philadelphia, 1977. C271 
[3] G. E. Karniadakis, M. Israeli, and S. A. Orszag. High-order splitting methods for the incompressible Navier-Stokes equations. J. Comput. Phys., 97(2):414-443, 1999. C272

[4] Y. Maday and A. T. Patera. Spectral element methods for the incompressible Navier-Stokes equations. In A. K. Noor and J. T. Oden, editors, State-of-the-Art Surveys on Computational Mechanics, chapter 3, pages 71-143, 1989. ASME. C275

[5] A. T Patera. A spectral element method for fluid dynamics: Laminar flow in a channel expansion. J. Comput. Phys., 54:468-488, 1984. C275

[6] A. Quarteroni and A. Valli. Domain Decomposition Methods for Partial Differential Equations. Oxford Science Publications, Oxford, 1999. C275

[7] G. Strang, and G. Fix. An Analysis of the Finite Element Method. Prentice-Hall, 1973. C274 